\title{
A New Method for Evaporation Modeling: Dynamic Evolving Neural-Fuzzy Inference System
}

\author{
Ozgur Kisi, ${ }^{1}$ Iman Mansouri, ${ }^{2}$ and Jong Wan $\mathrm{Hu}^{3,4}$ \\ ${ }^{1}$ School of Natural Sciences and Engineering, Ilia State University, Tbilisi, Georgia \\ ${ }^{2}$ Department of Civil Engineering, Birjand University of Technology, Birjand, Iran \\ ${ }^{3}$ Department of Civil and Environmental Engineering, Incheon National University, Incheon 22012, Republic of Korea \\ ${ }^{4}$ Incheon Disaster Prevention Research Center, Incheon National University, Incheon 22012, Republic of Korea \\ Correspondence should be addressed to Jong Wan Hu; jongp24@incheon.ac.kr
}

Received 8 May 2017; Revised 24 July 2017; Accepted 20 August 2017; Published 27 September 2017

Academic Editor: Francesco Viola

Copyright (c) 2017 Ozgur Kisi et al. This is an open access article distributed under the Creative Commons Attribution License, which permits unrestricted use, distribution, and reproduction in any medium, provided the original work is properly cited.

\begin{abstract}
Evaporation estimation is very essential for planning and development of water resources. The study investigates the ability of new method, dynamic evolving neural-fuzzy inference system (DENFIS), in modeling monthly pan evaporation. Monthly maximum and minimum temperatures, solar radiation, wind speed, and relative humidity data obtained from two stations located in Turkey are used as inputs to the models. The results of DENFIS method were compared with the classical adaptive neural-fuzzy inference system (ANFIS) by using root mean square error (RMSE), mean absolute relative error (MARE), and Nash-Sutcliffe Coefficient (NS) statistics. Cross validation was applied for better comparison of the models. The results indicated that DENFIS models increased the accuracy of ANFIS models to some extent. RMSE, MARE, and NS of the ANFIS model were increased by $11.13,11.45$, and $6.83 \%$ for the Antalya station and 20.11, 12.94\%, and 8.29\% for the Antakya station using DENFIS.
\end{abstract}

\section{Introduction}

One of the major issues that may negatively affect the agriculture of dry regions such as Iran is the evaporation of water resources. It has been estimated that evaporation is responsible for annual loss of more than $40 \%$ of water resources. Such loss of water may severely undermine not only the productivity of agricultural sector but environmental projects as well. The pan evaporation tests are prone to multiple errors originating from pan size and material, water depth in the pan, sun and wind exposure, and animal activity in the vicinity. The factors effectively deciding the degree of evaporation in given area are air and soil temperature, relative humidity, sunshine and wind speed, vapor pressure deficit, and atmospheric pressure. Thus, empirical evaporation prediction formulas $[1,2]$ need to account for several climatic factors associated with this phenomenon. However, the large amount of data required to develop such complex empirical relationships restricts their actual use in the field [3].

Evaporation pans are valuable in hydrology since they are simple strong instruments that incorporate the important physical factors, in particular radiation, temperature, humidity, and wind speed, into a single measure of evaporative demand $[4,5]$. However, it is unfeasible to place evaporation pans at each point where there is an arranged or existing reservoir and irrigation. It is additionally exceedingly improbable to have in distant areas where exact instruments can not be set up. A practical means of predicting the pan evaporation where no pans are accessible is of impressive noteworthiness to the hydrologists, meteorologists, and agriculturists [6].

Research has shown the artificial intelligence methods such as ANFIS and ANN to be very successful in civil engineering and especially in water resources analysis applications [7-16]. ANFIS, for example, was successfully used by Terzi et al. [13] to analyze the daily meteorology data of the Lake Egirdir in Turkey. In a study by Moghaddamnia et al. [11], pan evaporation was modeled by both ANFIS and artificial neural network (ANN) and their results showed only a slight difference between these methods. In a study by Shiri et al. [12], ANFIS was used to estimate the daily pan evaporation in Illinois (USA) and reported accurate 
estimations especially when using only a limited number of climatic parameters. In a study by Sanikhani et al. [3], ANFIS with grid partitioning (GP) and ANFIS with subtractive clustering (SC) were used to model the daily pan evaporation based on air temperature, wind speed, solar radiation, and relative humidity. Ultimately, this study reported that both ANFIS methods have the same degree of accuracy, which is better than the accuracy of multivariate nonlinear regression (MNLR), ANN, Stephens-Stewart (SS), and Penman [2] models. In a study by Goyal et al. [8], ANFIS was used to estimate the pan evaporation in subtropical climates, and more specifically in Karso watershed in India. Given the good accuracy of soft computing models, the majority of studies in this field have used these models for predicting the daily pan evaporation. In a study by Shirsath and Singh [17], the ANN and regression based models were used to estimate daily pan evaporation and compared with the multiple linear regression. In a study by Chang et al. [18], the self-organizing map neural network and back propagation neural network were compared in estimating daily pan evaporation. In a study by Furuhashi et al. [19], the suitability of hybridizing the Cuckoo optimization algorithm with ANN and ANFIS methods was tested for estimating daily evaporation. In a study by Lin and Lee [20], two data-driven methods, ANN, co-active neurofuzzy inference system, and multiple linear regression were applied to simulate daily evaporation at Pantnagar, India. In a study by Lughofer [21], radial basis neural networks and ANFIS approaches were utilized for multi-lead ahead prediction of evaporation from Layang reservoir, located in the southeast part of Malaysia. According to the authors' knowledge, the accuracy of dynamic evolving neural-fuzzy inference system has not been previously investigated in modeling pan evaporation.

In the present study, a new method, dynamic evolving neural-fuzzy inference system (DENFIS), was applied for evaporation modeling. This is the first study that uses DENFIS for solving this problem.

\section{Materials and Methods}

2.1. Case Study. In the current study, monthly maximum and minimum temperatures, solar radiation, wind speed, relative humidity, and pan evaporation data measured in Antalya (latitude $36.42^{\circ} \mathrm{N}$, longitude $30.44^{\circ} \mathrm{E}$, and altitude $64 \mathrm{~m}$ ) and Antakya (latitude $36.33^{\circ} \mathrm{N}$, longitude $36.30^{\circ} \mathrm{E}$, and altitude $100 \mathrm{~m}$ ) stations located in Mediterranean Region of Turkey were used (Figure 1). According to the Köppen criteria, the Mediterranean climate is identified by hot, dry, sunny summers and rainy winter season (Csa, Csb) and this is actually very different from the monsoon climate [22]. The climate is defined by mild and rainy winters with warm and dry summers. This condition is because of the air mass occupying the region. Actually, most of the heavy rainfall during the summer periods is related to the maritime tropical air mass. This air mass originates from the Atlantic Ocean. Also, the maritime polar air mass that comes from Western Europe is the other reason of the heavy rainfalls which cause the storms and severe floods. This phenomenon leads to a

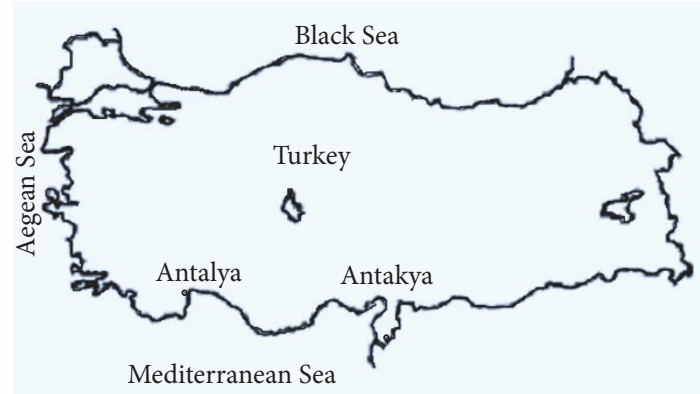

Figure 1: The location of the Antalya and Antakya stations.

severe damage to the agricultural land and green houses. In the Mediterranean zone, the summer season is subjected to the only maritime tropical and continental tropical air masses which causes a rainless summer. The average annual precipitation changes between 800 and 2000 millimeters. The frontal activities, exposure, the direction of mountain ranges, and altitude are some causes of the distribution of the precipitation. The Taurus Mountains prevent the fronts coming from the Mediterranean Sea. Therefore, the southwest slopes of the mountain are the rainy area of the region. Also, the annual precipitation in the southern high slopes of the Taurus Mountains increases up to 1000 millimeters because of orographic rainfall and decrease of yearly precipitation in shadow areas less than 500 millimeters in the poljes occurring in the vicinity of Antalya, for instance. At this area, rainfall begins at the beginning of autumn to the early month of the spring season. It means half of the total annual precipitation is related to the winter season. The average relative humidity is over $60 \%$. Also, because of the decrease in temperature in the late night and early morning particularly in hot summer days, the relative humidity exceeds $90 \%$. Today's dew and fog happen along the coastal belt. A reduction in evapotranspiration is caused due to high relative moisture causes. That is why the influence of the drought is lower than other components of the Mediterranean coastal areas, because of high moisture content [22].

Data used in the present study include 203 monthly values from 1983 to 2010 for the Antakya and 362 values from 1967 to 2006 for the Antalya station. Whole data were divided into three equal subsets and each subset was used for testing the applied models. Basic statistical properties of the used data are provided for each data set in Table 1. For Antalya station, the least evaporation value belongs to 1st data set while the 3rd data set includes the highest evaporation. In Antakya station, the lowest and highest values correspond to 1st and 2nd data sets, respectively; Antalya data show more scattered distribution compared to Antakya.

2.2. Dynamic Evolving Neural-Fuzzy Inference System (DENFIS). The principles of evolving neural networks and in particular evolving neurofuzzy method were first introduced by Kasabov and Song [23]. An important outcome of efforts of Kasabov and others in this field is the dynamic evolving neural-fuzzy inference system (DENFIS) [23], which is an 
TABLE 1: The monthly statistical parameters of pan evaporation data sets.

\begin{tabular}{|c|c|c|c|c|c|}
\hline Data set & $x$ mean, mm & $\mathrm{Sx}, \mathrm{mm}$ & $\operatorname{Cs} x$ & $x \mathrm{~min}, \mathrm{~mm}$ & $x \max , \mathrm{mm}$ \\
\hline \multicolumn{6}{|c|}{ Antalya } \\
\hline 1967 Jan-1985 Dec & 4.95 & 2.45 & 0.66 & 1.3 & 11.0 \\
\hline 1986 Jan-1995 Dec & 5.18 & 2.51 & 0.51 & 1.7 & 11.4 \\
\hline 1996 Jan-2006 Nov & 5.78 & 2.81 & 0.40 & 1.5 & 12.4 \\
\hline \multicolumn{6}{|c|}{ Antakya } \\
\hline 1983 Sep-1989 Dec & 4.21 & 2.19 & 0.09 & 0.9 & 8.1 \\
\hline 1990 Jan-2002 Nov & 4.39 & 2.30 & 0.28 & 1.1 & 9.8 \\
\hline 2003 Apr-2010 Sep & 5.27 & 1.75 & -0.40 & 1.6 & 7.9 \\
\hline
\end{tabular}

$x$ mean, $S x, C s x, x \min$, and $x \max$ indicate the overall mean, standard deviation, and skewness, respectively.

extension of Evolving Fuzzy Neural Network (EFuNN), itself based on the idea of developing a connectionist model in Evolving Connectionist System (ECOS) architecture [23]. Even before its development into DENFIS, EFuNN was known to have extensive ability to refine the model structure via an incremental sample-wise lifelong learning process by creating new structural components in a dynamic manner by using its five different input layers.

Input and output neurons can be fuzzified by a fuzzy quantization approach. Thus, fuzzy neural networks can be seen as connectionist structures with rules expressed with fuzzy logic $[19,20]$. These networks also possess all attributes of traditional neural networks such as recall, reinforcement, and hidden layers. Neurofuzzy inference systems are defined as the systems that employ fuzzy rules and associated fuzzy inference mechanisms for learning and rule optimization purposes. ANFIS is an example of this datacentered approach to learning. In DENFIS, the described model architecture is implemented in an evolving approach based on cross-linking to Takagi-Sugeno fuzzy systems.

The notable difference of this approach from other evolving fuzzy systems is the method of making a prediction for a new sample. In this respect, DENFIS follows a modelbased lazy learning approach, where network assesses the position of the input vector in the feature space, and forms, accordingly, a fuzzy inference system for predicting the output through a dynamic process based on the nearest fuzzy rules created during the incremental learning. In other words, the classical lazy learning of this network employs a samplebased approach, where local samples taken from the area closest to the query point are used to construct a small local model on demand [21]. This learning process is involved with the following synergies:

(1) Use of a fuzzy model with Takagi-Sugeno architecture: this means converting the model to a neurofuzzy format in a way that produces the same learning problem, which means linear consequent, nonlinear antecedent parameters, rules, or neurons need to be evolved on demand.

(2) Use of a clustering-based process for evolution of rules or neurons (evolving a new cluster means evolving a new rule).

(3) Local learning of consequent parameters.
The notable differences of this method from others include the use of ECM as Evolving Clustering Method, use of triangular membership functions (in place of Gaussian functions) as fuzzy sets, and the use of an alternative weighting scheme for local learning of consequent parameters.

DENFIS uses Takagi-Sugeno type fuzzy inference engine [24]. Such inference engine consists of fuzzy rules inferred in

$$
\begin{aligned}
& \text { if } x_{1} \text { is } R_{12}, x_{2} \text { is } R_{12}, \ldots, x_{q} \text { is } R_{1 q} \\
& \text { then } y \text { is } f_{1}\left(x_{1}, x_{2}, \ldots, x_{q}\right) \\
& \text { if } x_{1} \text { is } R_{21}, x_{2} \text { is } R_{22}, \ldots, x_{q} \text { is } R_{2 q} \\
& \text { then } y \text { is } f_{2}\left(x_{1}, x_{2}, \ldots, x_{q}\right) \\
& \quad \vdots \\
& \text { if } \quad x_{1} \text { is } R_{m 1}, x_{2} \text { is } R_{m 2}, \ldots, x_{q} \text { is } R_{m q} \\
& \text { then } y \text { is } f_{m}\left(x_{1}, x_{2}, \ldots, x_{q}\right),
\end{aligned}
$$

in which " $x_{j}$ is $R_{i j}$ ", $i=1,2, \ldots, m$, and $j=1,2, \ldots, q$ are $m \times q$ fuzzy arguments as $m$ prior defined form $m$ fuzzy rules. $x_{j} ; j=1,2, \ldots, q$ are prior parameters defined over universes of discussion. $x_{j} ; j=1,2, \ldots, q$ and $R_{i j}$; $i=1,2, \ldots, m$ and $j=1,2, \ldots, q$ are fuzzy arrangements determined by fuzzy membership functions $\mu_{R_{i j}} ; X_{j} \rightarrow$ $[0,1] ; i=1,2, \ldots, m$, and $j=1,2, \ldots, q$. The parameter $y$ is a resultant variable, and polynomial functions $f_{i} ; i=$ $1,2, \ldots, m$ are used. All fuzzy membership functions are triangular functions in DENFIS models. The membership functions are given as

$$
\mu(x)=m f(x, a, b, c)= \begin{cases}0 & x \leq a \\ \frac{x-a}{b-a} & a \leq x \leq b \\ \frac{c-x}{c-b} & b \leq x, c \\ 0 & c \leq x,\end{cases}
$$

in which $b$ denotes the cluster center on the $x$ dimension, $a=$ $b-d \times$ Dthr, and $c=b+d \times$ Dthr, $d=1.2$ to 2 ; here Dthr is a clustering variable. 
A zero-order Takagi-Sugeno type fuzzy inference system is defined as a system where resultant functions are crisp fixed parameters; that is, $f_{i}\left(x_{1}, x_{2}, \ldots, x_{q}\right)=C_{i} ; i=$ $1,2, \ldots, m$. If $f_{i}\left(x_{1}, x_{2}, \ldots, x_{q}\right), i=1,2, \ldots, m$, are linear functions, the system is named a first-order Takagi-Sugeno type fuzzy inference system. Similarly, it is named high-order Takagi-Sugeno fuzzy inference system if these functions are nonlinear.

The conclusion of inference of the output, $y^{0}$, for the input vector $x^{0}=\left[x_{1}^{0}, x_{2}^{0}, \ldots, x_{q}^{0}\right]$ is the weighted mean of each rule's output pointed out as follows [25]:

$$
y^{0}=\frac{\sum_{i=1}^{m} w_{i} f_{i}\left(x_{1}^{0}, x_{2}^{0}, \ldots, x_{q}^{0}\right)}{\sum_{i=1}^{m} w_{i}} .
$$

Here: $w_{i}=\prod_{j=1}^{q} \mu R_{i j} ; i=1,2, \ldots, m, j=1,2, \ldots, q$.

2.3. Adaptive Neurofuzzy Inference System. ANFIS is a multilayer feed-forward neurofuzzy network capable of combining the linguistic flexibility of fuzzy logic with the numeric capabilities of artificial neural networks (ANNs). Desirability of ANFIS lies in its ability to synergize the merits of ANN and fuzzy logic to map an input space to an output space more efficiently than either approach and achieve more effective forecasting models through its enhanced learning and data classification capabilities. Given the outstanding ability of ANFIS to infer fuzzy rules or expert knowledge from numerical data, this technique has found countless applications in classification, rule-based process control, and pattern recognition, for example, to analyze and predict the wind speed, dynamic load of power systems, and faults in engines. In a way similar to ANN's mechanism of solving function estimation problems, before making any prediction, ANFIS model needs to undergo a training phase specific to the data at hand and the target application [26, 27]. Theoretically, option variables can serve as the sole basis of development of nonparametric option pricing models with predictive and nonlinear modeling methods; but the use of human expert knowledge and decisions can significantly contribute to quality and speed of this process. Thus, an ideal prediction model is the one where option variables and human expert decisions and knowledge are all incorporated into the process. Such need makes ANFIS a perfectly suitable tool for merging the human expert knowledge and decisions and the option variables for better training and learning and thus better modeling of option prices.

From the functional perspective, ANFIS architecture is, on the one hand, an equivalent of fuzzy model as defined by Takagi-Sugeno-Kang (TSK model) and, on the other hand, a rough equivalent of Radial Basis Function Networks (RBFNs). But equivalence of RBFN and TSK fuzzy model is subject to the following requirements [28]:

(a) For both models, the aggregation method used to extract the overall outputs must be the same (weighted average or weighted sum).

(b) There must be an equal number of activation functions and fuzzy IF-THEN rules. (c) For the rule bases consisting of several inputs, each activation function must be equal to a composite input membership function. This can be achieved by several methods, the simplest of which is to incorporate Gaussian membership functions with the same variance into the rule base, while using the algebraic product for the "AND" operation. The product of the Gaussian membership functions will yield a multidimensional Gaussian RBFN.

(d) The activation functions on the output of neurons should have the same functions as their corresponding fuzzy rules.

\section{Application and Results}

The DENFIS method was employed for estimating pan evaporation based on the climatic data of maximum and minimum temperatures, solar radiation, wind speed, and relative humidity. The results of the proposed model were compared with those of the classical ANFIS model. The employed models were evaluated by using three commonly applied comparison criteria, namely, root mean square error (RMSE), mean absolute relative error (MARE), and NashSutcliffe Coefficient (NS). The expressions of the applied statistics are

$$
\begin{aligned}
\mathrm{RMSE} & =\sqrt{\frac{1}{n} \sum_{i=1}^{n}\left(E_{M, i}-E_{o, i}\right)^{2}}, \\
\mathrm{MARE} & =\frac{1}{n} \sum_{i=1}^{n} \frac{\left|E_{M, i}-E_{o, i}\right|}{E_{o, i}} * 100, \\
\mathrm{NS} & =1-\frac{\sum_{i=1}^{n}\left(E_{M, i}-E_{o, i}\right)}{\sum_{i=1}^{n}\left(E_{o, i}-\bar{E}_{o}\right)},
\end{aligned}
$$

where $E_{o, i}$ and $E_{M, i}$, respectively, are the observed and modeled pan evaporation at the $i$ th time step, $n$ is number of time steps, and $\bar{E}_{o}$ is mean of the observed pan evaporation.

First, whole data set was divided into three equal parts. Two parts were used in training of the applied models while the remaining one part was used for testing. Thus, three applications were obtained for each method. Then, the models were compared with each other according to the mean of the used statistics. Table 2 reports the periods of training and test data sets used for each DENFIS and ANFIS model. The accuracy of DENFIS models is mostly dependent on the choice of distance threshold value (Dthr) [29] and, therefore, different Dthr values were tried to find the optimal ones.

The optimal Dthr values obtained for the DENFIS models are $0.02,0.01$, and 0.013 for the M1, M2, and M3 data sets, respectively. For the ANFIS models, different number of membership functions (MFs) were tried and 2 Gaussian MFs for each input provided the best accuracy. 100 iterations were used for each model following the suggestions of [30]. 
TABLE 2: The training and test data sets used for each model.

\begin{tabular}{lcr}
\hline Cross validation & Training data set & Test data set \\
& Antalya & 1996 Jan-2006 Nov \\
M1 & 1967 Jan-1995 Dec & 1967 Jan-1985 Dec \\
M2 & 1986 Jan-2006 Nov & 1986 Jan-1995 Dec \\
M3 & Antakya & 2003 Apr-2010 Sep \\
& 1967 Jan-1985 Dec and 1996 Jan-2006 Nov & 1983 Sep-1989 Dec \\
M1 & 1983 Sep-2002 Nov & 1990 Jan-2002 Nov \\
M2 & 1990 Jan-2010 Sep & 1983 Sep-1989 Dec and 2003 Apr-2010 Sep \\
\hline
\end{tabular}

TABLE 3: Comparison of the DENFIS and ANFIS models in modeling pan evaporation-Antalya station.

\begin{tabular}{|c|c|c|c|c|}
\hline \multirow{2}{*}{ Statistics } & \multirow{2}{*}{ Cross validation } & \multirow{2}{*}{ Test data set } & \multicolumn{2}{|c|}{ Method } \\
\hline & & & DENFIS & ANFIS \\
\hline \multirow{4}{*}{ RMSE (mm) } & M1 & 1996-2006 & 0.976 & 0.962 \\
\hline & M2 & 1967-1985 & 1.036 & 1.483 \\
\hline & M3 & 1986-1995 & 0.862 & 0.788 \\
\hline & & Mean & 0.958 & 1.078 \\
\hline \multirow{4}{*}{ MARE (\%) } & M1 & 1996-2006 & 15.96 & 14.46 \\
\hline & M2 & 1967-1985 & 20.50 & 30.34 \\
\hline & M3 & 1986-1995 & 15.27 & 13.62 \\
\hline & & Mean & 17.24 & 19.47 \\
\hline \multirow{4}{*}{ NS } & M1 & 1996-2006 & 0.878 & 0.882 \\
\hline & M2 & 1967-1985 & 0.820 & 0.631 \\
\hline & M3 & 1986-1995 & 0.881 & 0.901 \\
\hline & & Mean & 0.860 & 0.805 \\
\hline
\end{tabular}

Comparison of the DENFIS and ANFIS models is made in Table 3 for modeling pan evaporation of Antalya station. According to the mean of the comparison statistics, the DENFIS has a better accuracy than the ANFIS model. Both methods have the highest accuracy for M3 data set while the M2 provides the worst results. The worst accuracy of the M2 models may be due to the highest skewness of this data set (see Table 1). The other reason may be the different data ranges between training and test data sets in M2. The minimum of the test data set $(1.3 \mathrm{~mm})$ is lower than that of the training period $(1.5 \mathrm{~mm})$ and this may cause some extrapolation difficulties for the applied models in estimating low pan evaporation values in the test phase. The DENFIS increased the accuracy of ANFIS by 11.13, 11.45, and $6.83 \%$ with respect to RMSE, MARE, and NS, respectively. Figure 2 illustrates the observed and estimated pan evaporation of Antalya by DENFIS and ANFIS in the test period. The ANFIS has higher $R^{2}$ than the DENFIS for the M1 and M3 data sets. For the DENFIS, however, the slope of the fit lines is closer to 1 than those of the ANFIS in case of M1 and M3. For the M2 data set, the DENFIS seems to be more successful than the ANFIS from the fit line coefficients and $R^{2}$ viewpoints.

Comparison of the DENFIS and ANFIS in estimating pan evaporation of Antakya station is made in Table 4. The optimal Dthr values obtained for the DENFIS models are $0.017,0.02$, and 0.013 for the M1, M2, and M3 data sets, respectively. It is clear from the table that both applied models give the worst result for the M3 data set. Here, also the main reason might be the fact that the maximum pan evaporation value $(9.8 \mathrm{~mm})$ of the test period is higher than the corresponding value $(8.1 \mathrm{~mm})$ of the training period for the $\mathrm{M} 3$ case. This gives the applied models some extrapolation difficulties in catching high pan evaporation values in the test stage. The RMSE, MARE, and NS accuracies of the ANFIS model were increased by $20.11,12.94$, and $8.29 \%$ using DENFIS model, respectively. The scatterplot comparison of both the methods is made in Figure 3 for the test stage. It is apparent from the figure that the slope and bias coefficients of the fit line equations are closer to those of the exact line ( $y=x$, slope: 1 and bias: 0 ) with a higher $R^{2}$ for the DENFIS model compared to those of the ANFIS in all three cases.

The results of the DENFIS and ANFIS models are tested by using one-way analysis of variance (ANOVA) and reported in Table 5. It is obvious from the table that the DENFIS models generally yield small testing values with higher significance levels for the ANOVA. It can be said that the DENFIS is more robust in pan evaporation estimation than the ANFIS. Recently, Kisi [31] modeled pan evaporation of Mersin and 

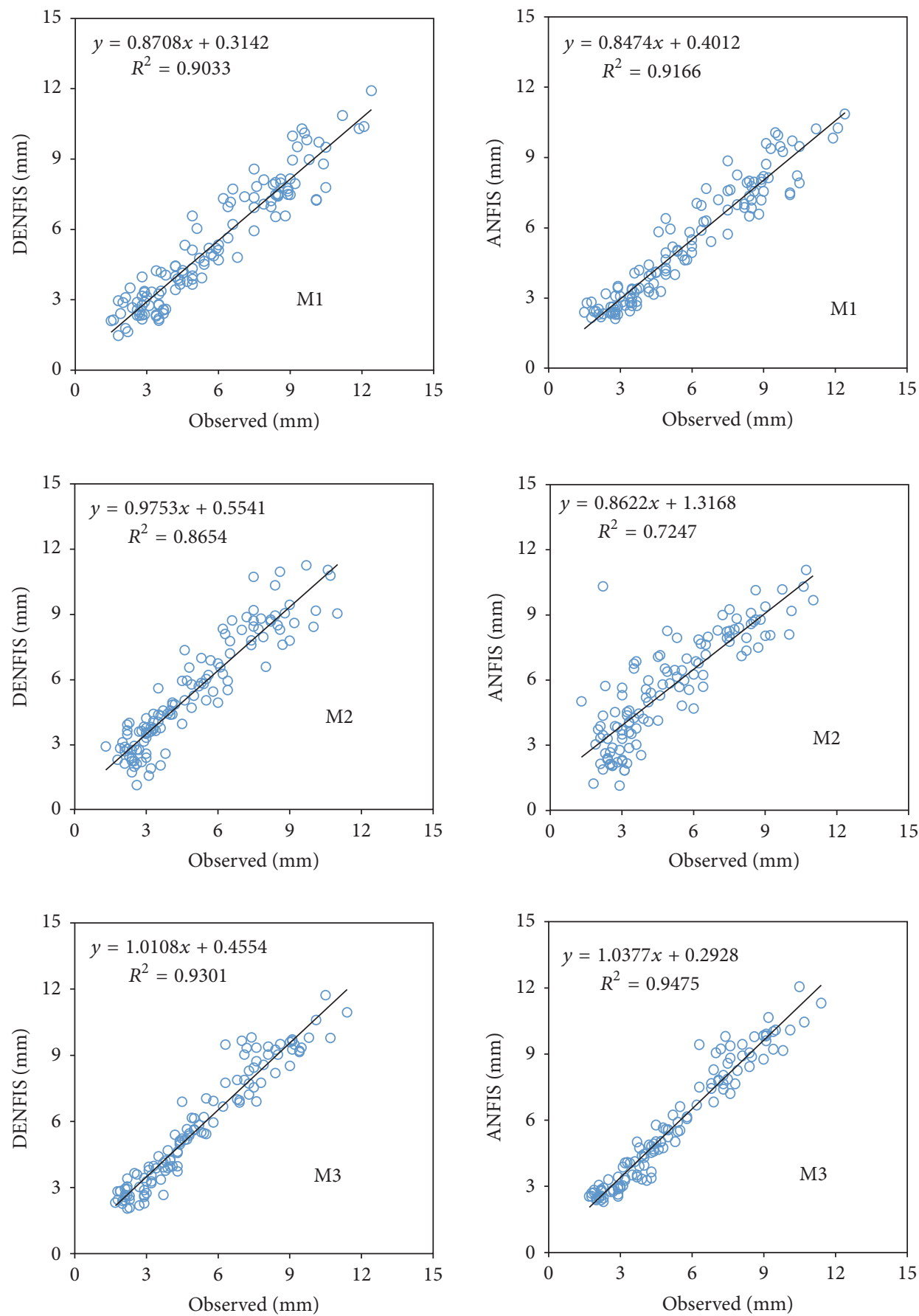

FIGURE 2: The observed and estimated pan evaporation using DENFIS and ANFIS methods for the M1, M2, and M3 data sets-Antalya.

Antalya stations which are located in Mediterranean Region of Turkey by using least square support vector regression (LSSVR), multivariate adaptive regression spline (MARS), and M5 model tree (M5Tree) methods and he found mean RMSE values as $0.904,1.013$, and $1.190 \mathrm{~mm}$ for the LSSVR, MARS, and M5Tree models in Mersin Station while corresponding values were $0.808,0.996$, and $0.859 \mathrm{~mm}$ in Antalya station, respectively. By comparing these results with those given in Tables 3 and 4, it can be said that the DENFIS model provided satisfactory estimates of the pan evaporation for the both stations.

\section{Conclusions}

This study investigated the accuracy of DENFIS as a new method in pan evaporation modeling. Monthly climatic data obtained from two stations from Turkey were used for testing 

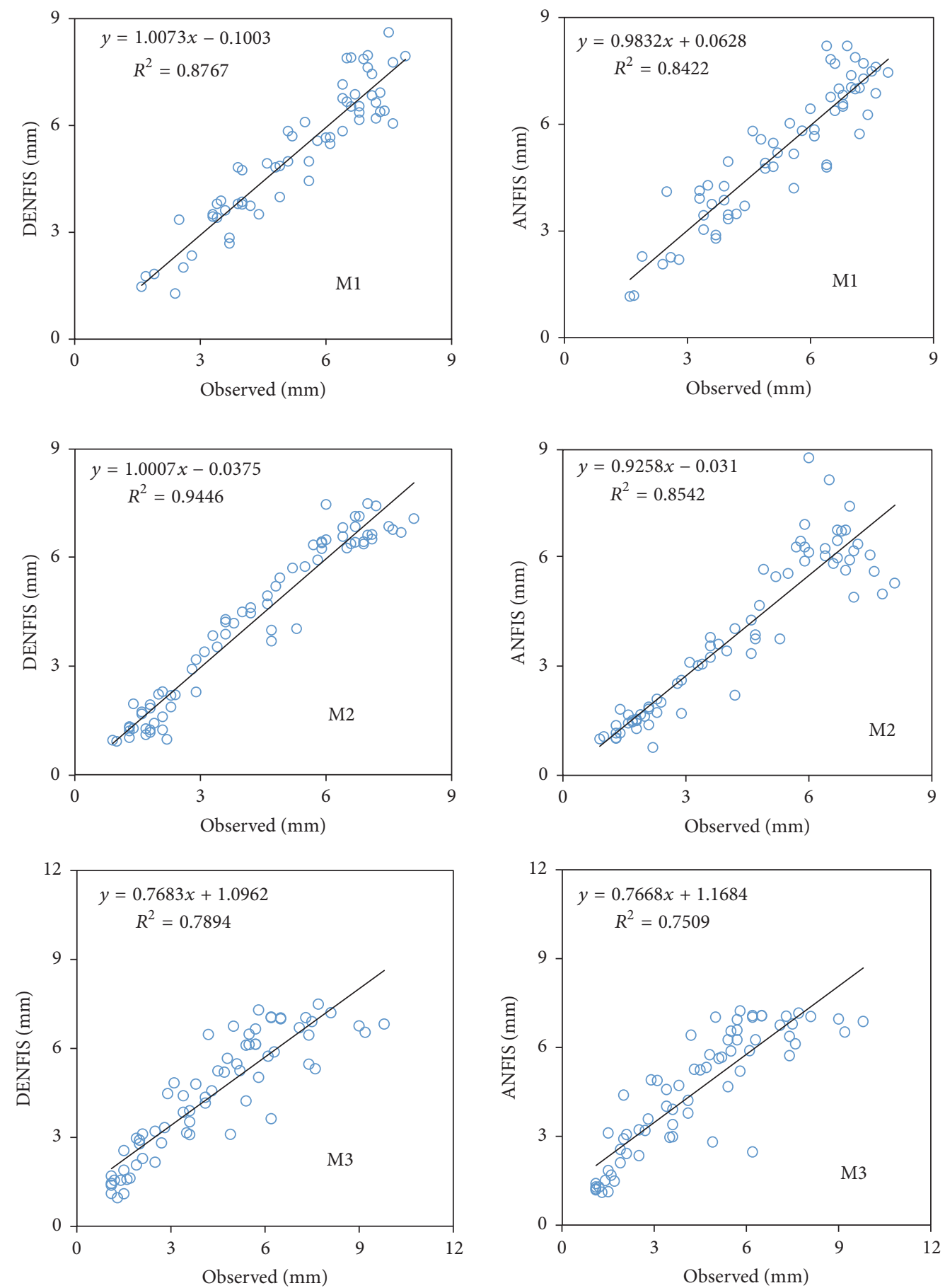

FIGURE 3: The observed and estimated pan evaporation using DENFIS and ANFIS methods for the M1, M2, and M3 data sets-Antakya.

the used models. Results were compared with those of the classic ANFIS models by using cross validation and RMSE, MAE, and NS statistics. According to the obtained results DENFIS model improved the ability of ANFIS by 11.13, 11.45, and $6.83 \%$ from the viewpoints of RMSE, MARE, and NS, respectively, for Antalya station, while, in Antakya station, the ANFIS accuracy was improved by $20.11 \%$ for RMSE, $12.94 \%$ for MARE, and $8.29 \%$ for NS. ANOVA test results indicated the superior accuracy of the DENFIS to the ANFIS models. Comparison with previous studies suggested the use of this method (DENFIS) in pan evaporation modeling.

The ability of DENFIS was compared with ANFIS method using two stations' data. The proposed method can also be compared with other data-driven methods in modeling 
TABLE 4: Comparison of the DENFIS and ANFIS models in modeling pan evaporation-Antakya station.

\begin{tabular}{|c|c|c|c|c|}
\hline \multirow{2}{*}{ Statistics } & \multirow{2}{*}{ Cross validation } & \multirow{2}{*}{ Test data set } & \multicolumn{2}{|c|}{ Method } \\
\hline & & & DENFIS & ANFIS \\
\hline \multirow{4}{*}{ RMSE (mm) } & M1 & $2003-2010$ & 0.659 & 0.740 \\
\hline & M2 & 1983-1989 & 0.528 & 0.914 \\
\hline & M3 & 1990-2002 & 1.054 & 1.152 \\
\hline & & Mean & 0.747 & 0.935 \\
\hline \multirow{4}{*}{ MARE (\%) } & M1 & 2003-2010 & 10.76 & 12.60 \\
\hline & M2 & 1983-1989 & 12.15 & 14.93 \\
\hline & M3 & 1990-2002 & 20.29 & 22.08 \\
\hline & & Mean & 14.40 & 16.54 \\
\hline \multirow{4}{*}{ NS } & M1 & $2003-2010$ & 0.856 & 0.818 \\
\hline & M2 & 1983-1989 & 0.941 & 0.823 \\
\hline & M3 & 1990-2002 & 0.788 & 0.747 \\
\hline & & Mean & 0.862 & 0.796 \\
\hline
\end{tabular}

TABLE 5: Analysis of variance (ANOVA) for pan evaporation estimation in the test period.

\begin{tabular}{|c|c|c|c|c|}
\hline \multirow{2}{*}{ Method } & \multicolumn{2}{|c|}{ Antalya } & \multicolumn{2}{|c|}{ Antakya } \\
\hline & $F$ statistic & Resultant sig. level & $F$ statistic & Resultant sig. level \\
\hline \multicolumn{5}{|c|}{ DENFIS } \\
\hline M1 & 1.574 & 0.211 & 0.035 & 3.920 \\
\hline M2 & 1.784 & 0.183 & 0.009 & 0.926 \\
\hline M3 & 2.345 & 0.127 & 0.046 & 0.830 \\
\hline \multicolumn{5}{|c|}{ ANFIS } \\
\hline M1 & 2.008 & 0.158 & 0.006 & 0.938 \\
\hline M2 & 3.992 & 0.047 & 0.894 & 0.346 \\
\hline M3 & 2.098 & 0.149 & 0.152 & 0.697 \\
\hline
\end{tabular}

pan evaporation using more data having different climatic conditions.

\section{Conflicts of Interest}

The authors declare that they have no conflicts of interest.

\section{Acknowledgments}

This research was supported by Basic Science Research Program through the National Research Foundation of Korea (NRF) funded by the Ministry of Science, ICT \& Future Planning (2017R1A2B2010120).

\section{References}

[1] J. L. Monteith, "Evaporation and environment," Symposia of the Society for Experimental Biology, vol. 19, pp. 205-234, 1965.

[2] H. L. Penman, "Natural evaporation from open water, hare soil and grass," Proceedings of the Royal Society of London. Series A, Mathematical and physical sciences, vol. 193, no. 1032, pp. 120$145,1948$.

[3] H. Sanikhani, O. Kisi, M. R. Nikpour, and Y. Dinpashoh, "Estimation of daily pan evaporation using two different adaptive neuro-fuzzy computing techniques," Water Resources Management, vol. 26, no. 15, pp. 4347-4365, 2012.
[4] M. L. Roderick and G. D. Farquhar, "Changes in Australian pan evaporation from 1970 to 2002," International Journal of Climatology, vol. 24, no. 9, pp. 1077-1090, 2004.

[5] M. L. Roderick, L. D. Rotstayn, G. D. Farquhar, and M. T. Hobbins, "On the attribution of changing pan evaporation," Geophysical Research Letters, vol. 34, no. 17, Article ID L17403, 2007.

[6] O. Kisi, "Daily pan evaporation modelling using a neuro-fuzzy computing technique," Journal of Hydrology, vol. 329, no. 3-4, pp. 636-646, 2006.

[7] A. El-Shafie, H. M. Alsulami, H. Jahanbani, and A. Najah, "Multi-lead ahead prediction model of reference evapotranspiration utilizing ANN with ensemble procedure," Stochastic Environmental Research and Risk Assessment, vol. 27, no. 6, pp. 1423-1440, 2013.

[8] M. K. Goyal, B. Bharti, J. Quilty, J. Adamowski, and A. Pandey, "Modeling of daily pan evaporation in sub tropical climates using ANN, LS-SVR, fuzzy logic, and ANFIS," Expert Systems with Applications, vol. 41, no. 11, pp. 5267-5276, 2014.

[9] X. Li, H. R. Maier, and A. C. Zecchin, "Improved PMI-based input variable selection approach for artificial neural network and other data driven environmental and water resource models," Environmental Modelling and Software, vol. 65, pp. 15-29, 2015.

[10] X. Li, A. C. Zecchin, and H. R. Maier, "Selection of smoothing parameter estimators for general regression neural networks Applications to hydrological and water resources modelling," 
Environmental Modelling and Software, vol. 59, pp. 162-186, 2014.

[11] A. Moghaddamnia, M. Ghafari Gousheh, J. Piri, S. Amin, and D. Han, "Evaporation estimation using artificial neural networks and adaptive neuro-fuzzy inference system techniques," Advances in Water Resources, vol. 32, no. 1, pp. 88-97, 2009.

[12] J. Shiri, W. Dierickx, A. Pour-Ali Baba, S. Neamati, and M. A. Ghorbani, "Estimating daily pan evaporation from climatic data of the State of Illinois, USA using adaptive neuro-fuzzy inference system (ANFIS) and artificial neural network (ANN)," Hydrology Research, vol. 42, no. 6, pp. 491-502, 2011.

[13] Ö. Terzi, M. E. Keskin, and E. D. Taylan, "Estimating evaporation using ANFIS," Journal of Irrigation and Drainage Engineering, vol. 132, no. 5, pp. 503-507, 2006.

[14] I. Mansouri, A. Gholampour, O. Kisi, and T. Ozbakkaloglu, "Evaluation of peak and residual conditions of actively confined concrete using neuro-fuzzy and neural computing techniques," Neural Computing and Applications, pp. 1-16, 2016.

[15] I. Mansouri and O. Kisi, "Prediction of debonding strength for masonry elements retrofitted with FRP composites using neuro fuzzy and neural network approaches," Composites Part B: Engineering, vol. 70, pp. 247-255, 2015.

[16] I. Mansouri, T. Ozbakkaloglu, O. Kisi, and T. Xie, "Predicting behavior of FRP-confined concrete using neuro fuzzy, neural network, multivariate adaptive regression splines and M5 model tree techniques," Materials and Structures, vol. 49, no. 10, pp. 4319-4334, 2016.

[17] P. B. Shirsath and A. K. Singh, "A comparative study of daily pan evaporation estimation using $\mathrm{ANN}$, regression and climate based models," Water Resources Management, vol. 24, no. 8, pp. 1571-1581, 2010.

[18] F.-J. Chang, L.-C. Chang, H.-S. Kao, and G.-R. Wu, "Assessing the effort of meteorological variables for evaporation estimation by self-organizing map neural network," Journal of Hydrology, vol. 384, no. 1-2, pp. 118-129, 2010.

[19] T. Furuhashi, T. Hasegawa, S. Horikawa, and Y. Uchikawa, "An adaptive fuzzy controller using fuzzy neural networks," in Proceedings of the Fifth IFSA World Congress, Seoul, South Korea, 1993.

[20] C. Lin and C. Lee, Neuro Fuzzy Systems, Prentice Hall, Englewood Cliffs, NJ, USA, 1996.

[21] E. Lughofer, Evolving Fuzzy Systems - Methodologies, Advanced Concepts and Applications, Springer, India, 2011.

[22] P. T. Nastos, N. Politi, and J. Kapsomenakis, "Spatial and temporal variability of the Aridity Index in Greece," Atmospheric Research, vol. 119, pp. 140-152, 2013.

[23] N. K. Kasabov and Q. Song, "DENFIS: Dynamic evolving neural-fuzzy inference system and its application for time-series prediction," IEEE Transactions on Fuzzy Systems, vol. 10, no. 2, pp. 144-154, 2002.

[24] T. Takagi and M. Sugeno, "Fuzzy identification of systems and its applications to modeling and control," IEEE Transactions on Systems, Man and Cybernetics, vol. 15, no. 1, pp. 116-132, 1985.

[25] J. Sobhani and M. Najimi, "Numerical study on the feasibility of dynamic evolving neural-fuzzy inference system for approximation of compressive strength of dry-cast concrete," Applied Soft Computing Journal, vol. 24, pp. 572-584, 2014.

[26] M. Buragohain and C. Mahanta, "A novel approach for ANFIS modelling based on full factorial design," Applied Soft Computing, vol. 8, no. 1, pp. 609-625, 2008.
[27] F.-J. Chang and Y.-T. Chang, "Adaptive neuro-fuzzy inference system for prediction of water level in reservoir," Advances in Water Resources, vol. 29, no. 1, pp. 1-10, 2006.

[28] J. Jantzen, “Neurofuzzy Modelling," Tech. Rep., Technical University of Denmark, Department of Automation, Denmark, 1998.

[29] S. Heddam and N. Dechemi, "A new approach based on the dynamic evolving neural-fuzzy inference system (DENFIS) for modelling coagulant dosage (Dos): case study of water treatment plant of Algeria," Desalination and Water Treatment, vol. 53, no. 4, pp. 1045-1053, 2015.

[30] O. Kisi and H. Sanikhani, "Modelling long-term monthly temperatures by several data-driven methods using geographical inputs," International Journal of Climatology, vol. 35, no. 13, pp. 3834-3846, 2015.

[31] O. Kisi, "Pan evaporation modeling using least square support vector machine, multivariate adaptive regression splines and M5 model tree," Journal of Hydrology, vol. 528, pp. 312-320, 2015. 

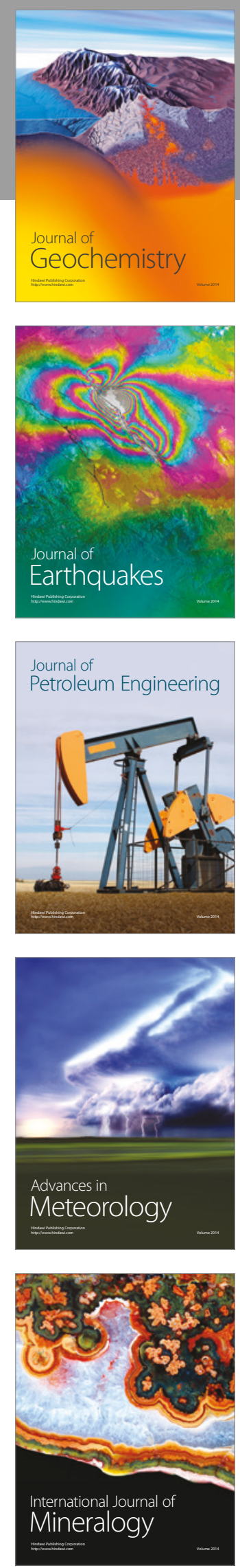
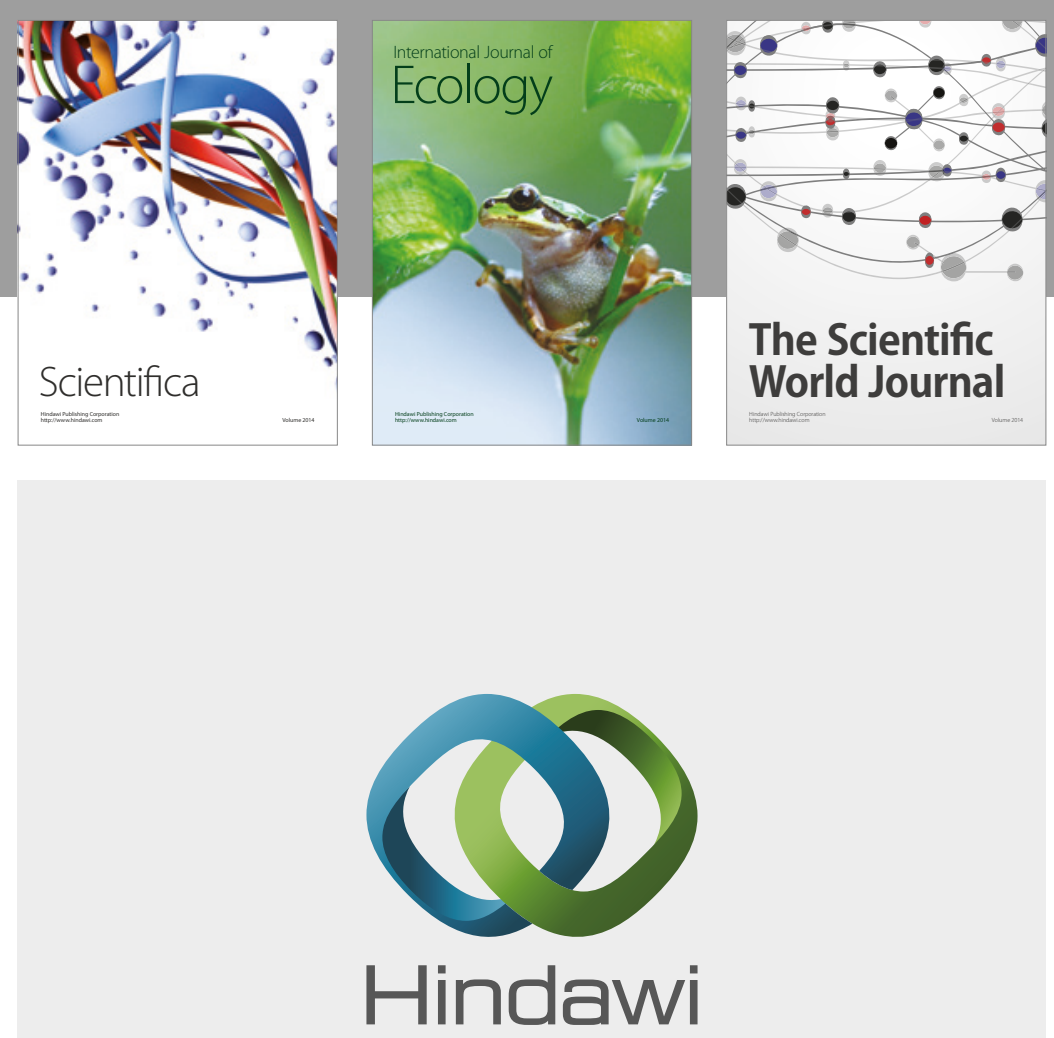

Submit your manuscripts at

https://www.hindawi.com
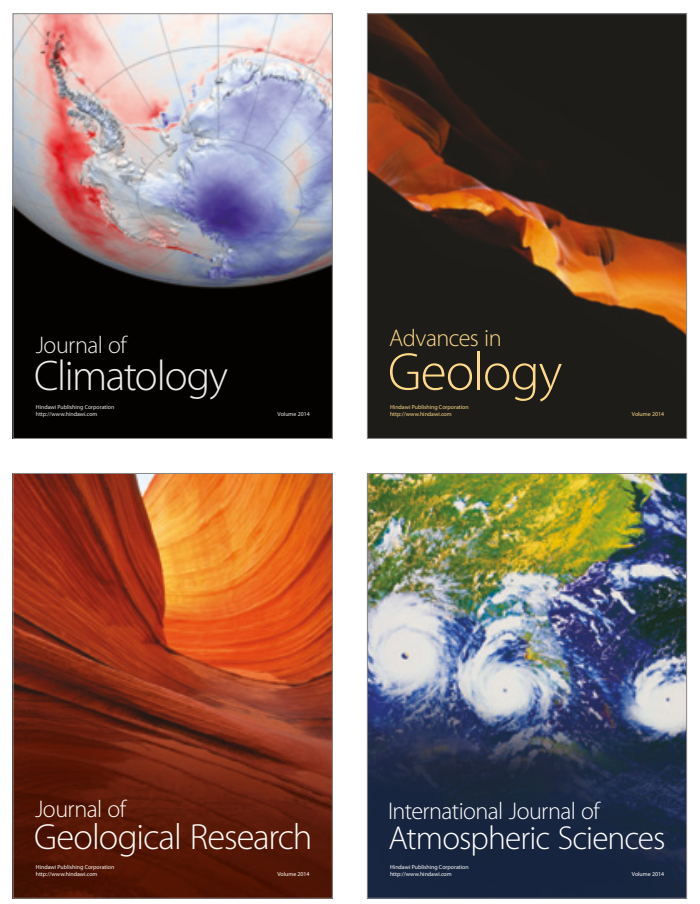

The Scientific

World Journal
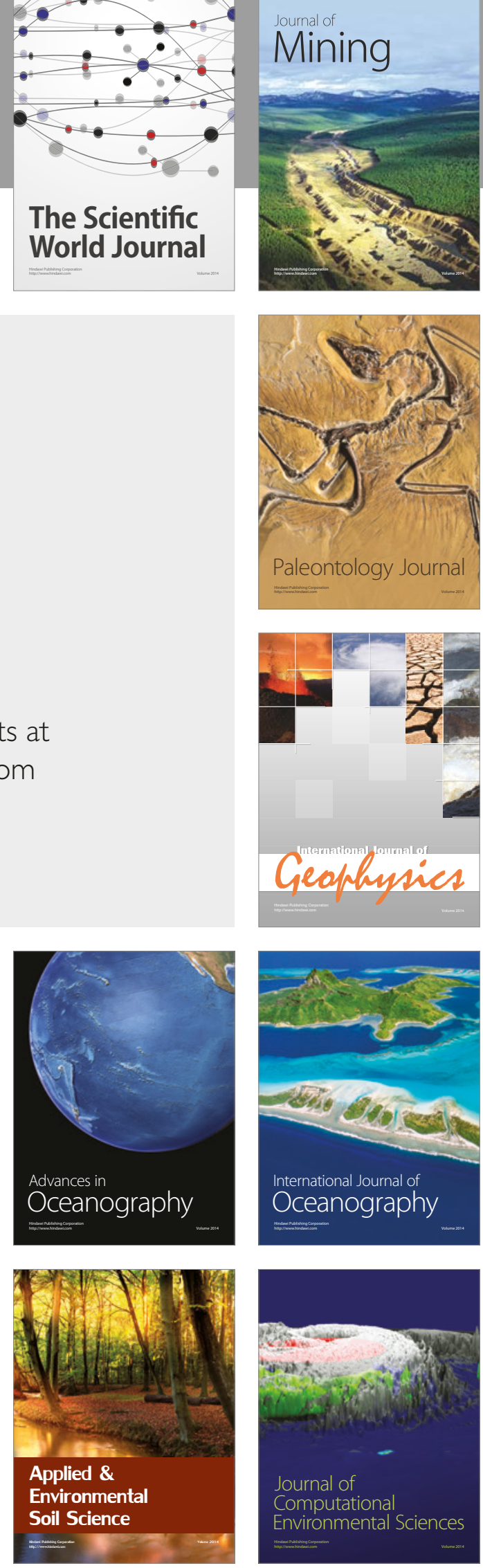